\title{
The effectiveness of oral zinc supplementation in patients with liver cirrhosis and hepatic encephalopathy
}

\author{
Zaur Orujov, MD \\ ${ }^{1}$ Medi Club Hospital
}

\begin{abstract}
The role of zinc in biochemical reactions in the human body and the need for sufficient intake of zinc have been known to science for long time. Zinc is a cofactor of more than 300 biochemical reactions, including those occurring in the liver. The human genome encodes about 3,000 zinc proteins. At least thirty proteins homeostatically control the vesicular accumulation and intracellular distribution of zinc, as well as the concentration of zinc ions (1). In $20 \%$ of patients with compensated and 50\% of patients with decompensated cirrhosis of the liver, both quantitative and qualitative nutritional problems are observed (2). There is still not much work and recommendations on the diet of patients with hepatic encephalopathy in the modern doctor's arsenal. A review of articles describing the effectiveness of adding zinc to the diet of patient with cirrhosis and hepatic encephalopathy, as we know, has not been conducted yet. The purpose of this review is a detailed analysis of the available scientific data on the effectiveness of zinc in patients with hepatic encephalopathy and, if it is possible, the development of specific recommendations for medical practitioners, as well as identification of perspective directions for further researches.
\end{abstract}

Keywords: hepatic encephalopathy, nutrition, zinc

\section{Method}

The literature search, analyzed in this review, was done by using databases as PubMed and Google Scholar.

Only clinical trials conducted in humans and was in compliance with ethical standards that are generally accepted for scientific work were selected for this review. All non-medical works were excluded out off more than 20000 ones. Of the remaining studies from 1984 to 2014 , only 8 were clinical and only 6 were RCTs. The total number of patients in our review is 312. 45 patients described in the review of research depending serum zinc and olfactory dysfunction in patients with cirrhosis and HE were not included in it, as they did not obtain any zinc supplement during the study, but were merely subjected of checking for level of serum zinc (9).

List of abbreviations:

RCT- randomized controlled trial

HE - hepatic encephalopathy

LOLA - 1-ornithine 1-aspartate

HRQOL - Health-Related Quality of Life

BCAA - Branched-chain amino acids

SF-36 - The Short Form-36

FS - Food supplement

\section{Results}

The first RCT to describe the effect of adding zinc to a patient's diet was a study by Reding, P. et al. in 22 patients with chronic cirrhosis, zinc deficiency and HE. Patients received $600 \mathrm{mg}$ of zinc acetate for 7 days demonstrated both an increase zinc level in serum and an improvement the results of the neuropsychological trailmaking test (3). It should be noted that $600 \mathrm{mg}$ of zinc acetate is 4.5 times higher than the Upper Tolerable Limit (4) (5).

Further, in 1991, a double-blind crossover study by Riggio O. et al. in 15 patients who received $600 \mathrm{mg}$ of zinc sulfate for 10 days showed no difference in blood ammonia levels, as well as in neuropsychological and neurophysiological tests between the zinc-treated group and the control group receiving a placebo. However, as well as previous researchers, they recorded a rise in serum zinc to values corresponding to the norm (6). In this study the daily zinc dose also exceeded the Upper Tolerable Limit, this time by 3.5 times (4) (5).

In 1993, Bresci G. with a group of scientists conducted a long study involving 90 patients with cirrhosis and HE, during which they compared the effectiveness of the long-term (6 months) usage of zinc acetate in a dose of $600 \mathrm{mg}$ with a background of standard PE therapy for that time (a diet containing $0.8 \mathrm{~g} / \mathrm{kg} / \mathrm{day}$ of protein and $90 \mathrm{~g} /$ day of lactulose). The control group received only standard therapy. Placebo control was not carried out.

To assess the degree of hepatic encephalopathy, both at the beginning and at the end of each treatment period, psychometric tests were used. The values of these tests were better in the zinc group, but the difference was not statistically significant.

At the same time, the patients who never showed clinical signs of hepatic encephalopathy during the trial were $88.6 \%$ in zinc-group and $86 \%$ in only standard therapy group (7). Considering the duration of receiving for 6 months, usage of zinc in such a big doze in this research is alarming. 
In 2000, long-term follow-up of 34 patients with cirrhosis, HE, and decreased level of serum zinc was conducted. All patients received zinc supplement (Unizink, ZinkaminFalk) relevantly to $30 \mathrm{mg}$ elemental zinc, as well as LOLA 6-9 g/d. Patients were not divided into groups. Each month laboratory tests were done. After normalization of serum zinc level, adding zinc supplement stopped. Patients whose serum zinc levels were falling were given zinc supplements again at the same dose. The evaluating criteria was the concentration of serum zinc and ammonia in plasma, as well as neuropsychological tests. During the many months of research, each time a decrease in the level of zinc in the blood accompanied with increase in the level of ammonia, and vice versa, when the level of zinc was restored, the level of ammonia fell.

In 2005, a small study was conducted that demonstrated a relationship between neuropsychological tests and olfactory dysfunction in 45 patients with cirrhosis and HE. However, the same study demonstrated that olfactory dysfunction in these patients was not associated with serum zinc levels (9).

In 2010, Y. Takuma et al. Conducted a study over 6 months in 79 patients, who were divided into 2 groups: the group receiving lactulose $(30-60 \mathrm{ml} /$ day) and BCAA (12 g / day) and the group receiving lactulose (30-60 ml / day), BCAA (12 g / day), and $225 \mathrm{mg}$ of Polaprezinc (containing $51 \mathrm{mg}$ of zinc and $174 \mathrm{mg}$ of 1 - carnosine). For the first time in selecting patients for a study of the effectiveness of adding zinc to the diet of patients withHE, topical West Haven Criteria that are relevant today were used. A study showed that prolonged use of $225 \mathrm{mg}$ of Polaprezinc improved HRQOL. As the criterion the SF-36 questionnaire was used (10).

In 2012, a team of scientists from Iran conducted a 3month randomized, double-blind, placebo-controlled study in 60 patients with non-alcoholic cirrhosis. Zinc group patients received $50 \mathrm{mg}$ of elemental zinc as zinc sulfate. The study showed an overall positive dynamics in patients. Particularly, after three months the average Child-Pugh score significantly decreased in the zinc group, while in the placebo group it increased slightly. The authors suggest that this result can also positively affect the flowing of HE, however, no specific tests were performed as part of the study (11).

Finally, in 2014, the first full-fledged, randomized, double-blind, placebo-controlled study, which showed a 3 -month positive result of using $150 \mathrm{mg}$ of elemental zinc (in the form of acetate) in the correction of elevated levels of ammonia in the blood of patients with liver cirrhosis and HE appeared. The study demonstrated the effectiveness of zinc to reduce elevated levels of ammonia in the blood of patients with cirrhosis and HE. Also, despite the fact that $150 \mathrm{mg}$ of elemental zinc significantly exceeds the Upper Tolerable Limit of $40 \mathrm{mg}$ (4) (5), the zinc preparation showed a rather high level of safety (12).

\section{Discussion}

Probably, the positive effect of adding zinc to the diet of patients with $\mathrm{HE}$ is mediated by peripheral mechanisms of participation in lowering the level of ammonia in the blood (8). Did the reviewed research results give an answer to the main question of our review? Is the use of zinc effective in treating patients with cirrhosis and hepatic encephalopathy? To a certain extent, yes. We can be sure that the addition of zinc supplements to patients with cirrhosis and HE restores its concentration in serum. Studies also showed the effectiveness of zinc in lowering ammonia levels, in improving the life quality of patients. When we talk about the criteria for HE itself, the data are still contradictory and the problem here is not only in the presented studies, but also in the difficulty of diagnosing $\mathrm{HE}$ itself. Among the limitations of our study, it should be noted that it was conducted on only 312 patients. It included only one modern, full-fledged, randomized, double blind, placebo-controlled study. So far, not a single study has been conducted that would demonstrate the effect of zinc preparations on the survival of patients with $\mathrm{HE}$ against cirrhosis.

\section{Conclusion}

After analyzing clinical studies of the effectiveness of zinc in patients with cirrhosis and PE, considering the serious limitations of the evidence base on the one hand and the positive results of a number of primary studies, a high level of zinc safety even when exceeding the Upper Tolerable Limit (12) and the low cost of FS with zinc in another, we think it is acceptable and advisable to administer zinc to patients with cirrhosis, PE, increased levels of ammonia in the blood and decreased levels of serum zinc.

Future RCTs with a large number of patients should be carried out to clarify the results of previous studies.

Financial source: Did not use financial resources.

\section{Conflict of interest: None.}

\section{REFERENCES}

1. Ministry of Health (1954). Mortality and Morbidity During the London Fog of December 1952. London, HMSO.

2. Paul W. Environmental Epidemiology. 1st ed. Open 
University Press; 2006

3. Dockery DW, Pope CA et al. (1993). An association between air pollution and mortality in six U.S. cities. New England Journal of Medicine 329(24): 1753-9.

4. Piriyev Y, Gozalov S. Ecological Problems of Motor Transport of Azerbaijan. Proceedings REAL CORP 2012 Tagungsband 14-16 May 2012, Schwechat

5. Annual Emissions and Fuel Consumption for an "Average" Passenger Car. U.S. Environmental Protection Agency (1997 April). [Accessed 2013 March 21]. Available from: URL: http://msl1.mit.edu/EPA-average-ann-emit.pdf

6. Main causes of death among population. The State Statistics Committee of Azerbaijan Republic (2013). [Accessed 2013 March 21]. Available from: URL: http://www.stat.gov.az/source/healthcare/indexen.php

7. The Second Global Report on Road Safety. WHO (2013). [Accessed 2013 March 21]. Available from: URL:

http://www.who.int/violence_injury_prevention/road_ safety_status/2013/en/index.html 